\title{
Knowledge elaboration: A cognitive load perspective
}

\author{
Slava Kalyuga* \\ School of Education, University of New South Wales, Sydney, NSW 2052, Australia
}

\begin{abstract}
The process of knowledge elaboration is considered from the perspective of cognitive load theory. This theory assumes that the available knowledge structures in long-term memory (LTM) are used to organize and guide cognitive processing in complex learning. Accordingly, the role of external instructional guidance in the process of knowledge elaboration could be described as providing a substitute for knowledge structures missing from LTM. Thus, the executive guidance in complex learning environments is shared between the person (based on his/her LTM knowledge structures) and another expert or instructional means. This article analyzes instructional implications of this assumption. Adaptive learning environments are suggested for tailoring knowledge elaboration processes to changing characteristics of individual learners. Means for identifying and predicting the learner's LTM-based executive guidance are proposed so that they can be utilized in the building of adaptive learning environments.
\end{abstract}

(C) 2009 Elsevier Ltd. All rights reserved.

Keywords: Cognitive load theory; Executive guidance; Adaptive learning environments; Metacognition

\section{Introduction}

Knowledge elaboration is using prior knowledge to continuously expand and refine new material based on such processes as organizing, restructuring, interconnecting, integrating new elements of information, identifying relations between them, and relating the new material to the learner's prior knowledge. Knowledge elaboration processes result in knowledge components additional to those given in the task statement or instructional message by creating links between prior knowledge and the new information (Anderson, 1995; Mayer, 1984; Pressley, 1982; Reigeluth, Merrill, Wilson, \& Spiller, 1980). For example, the success in learning from worked examples depends on the quality of example elaborations (Stark, Mandl, Gruber, \& Renkl, 2002) or "selfexplanations" (Chi, Bassok, Lewis, Reinmann, \& Glaser, 1989). The quality of such elaborations could be measured by the proportion of deep principle-based rather than superficial elaborations. Knowledge elaboration processes are essential

\footnotetext{
* Corresponding author. Tel.: +61 29385 1985; fax: +61 293851946.

E-mail address: s.kalyuga@unsw.edu.au
}

for meaningful learning since they allow the learners to organize knowledge into a coherent structure and integrate new information with existing knowledge structures.

The present article considers knowledge elaboration from the perspective of cognitive load theory. This theory focuses on processing limitations of our cognitive system and means for managing these limitations in learning and instruction. Cognitive load theory assumes two key functional components of our cognitive architecture (see Sweller, 2003, 2004; Van Merriënboer \& Sweller, 2005, for theoretical foundations of the theory). One is a large and permanent store of organized information. The concept of long-term memory (LTM) and knowledge structures in LTM relate to this component.

The other basic component is a mechanism that limits the scope of immediate changes to the information store. The concept of working memory (WM) is central to this function. Some theories consider WM as a separate structural component of the information-processing system (Baddeley, 1986), while other models regard WM as an activated part of LTM (Cowan, 2001). The essential common attributes of most existing WM models are severe limitations in capacity and duration when consciously processing unfamiliar elements of information (for an overview, see Miyake \& Shah, 1999). 
If more than around seven unrelated chunks of new information are processed simultaneously, then WM is overloaded (Miller, 1956). This number drops further if there are interrelations between the chunks that also need to be processed in WM. These limitations result in relatively slow and incremental changes to LTM when this new information is organized and encoded into LTM.

Cognitive load theory also assumes that the available knowledge structures in LTM are essential for preventing WM overload and for guiding cognitive processes. Accordingly, the role of external instructional guidance in the process of knowledge elaboration could be described as providing a substitute for missing LTM structures. The following sections of the present article will analyze instructional implications of the above assumptions, in particular the need for tailoring knowledge elaboration processes to changing characteristics of individual learners. Moreover, means for identifying and predicting the learner's LTM-based executive guidance in real time based on the knowledge structures learners bring into the processing of tasks are proposed. These knowledge structures could be used in the building of adaptive learning environments.

\subsection{LTM knowledge structures and processing limitations of our cognitive system}

Despite the very limited capacity and duration of WM, we are nevertheless able to acquire a massive knowledge base in LTM, successfully function in diverse environments, cognitively organize complex situations, appropriately direct our attention, and coordinate different cognitive activities. Knowledge structures held in LTM allow us to effectively reduce WM load by encapsulating many elements of information into larger, higher-level units that could be treated as elements in WM (Chi, Glaser, \& Rees, 1982). The reduction of required processing resources could also be achieved by practicing the application of certain knowledge structures until they can operate under automatic rather than controlled conditions (Hasher \& Zacks, 1979; Kotovsky, Hayes, \& Simon, 1985; Shiffrin \& Schneider, 1977). Thus, the capacity limitations of WM can be bypassed to a certain extent by automated processes that pose very little demands on WM.

Major characteristics of organized knowledge structures in LTM are associated with the concept of schemas as generic cognitive constructs that are used for categorizing stable information patterns. Schemas abstract generalizations about some class of objects and procedures from specific instances, encode general categories, typical features and relationships (Anderson, 1977; Bartlett, 1932; Rumelhart, 1980). Schemas contain related concepts of declarative and/or procedural (e.g., procedural schemas such as strategies) nature including procedural information that facilitates action associated with a particular conceptual category. Schema-based knowledge could be more general or specific, linked together and organized into sophisticated hierarchical structures. As a cognitive unit, the schema represents a higher level of organization than a simple collection of lower-level components. Because a schema is treated as a single unit in WM, such high-level structures require less cognitive capacity for processing than the multiple lower-level elements they contain, thus making WM load more manageable.

In cognitive load theory, acquisition and automation of schemas in LTM are considered as the most significant factors in preventing cognitive overload in learning (Sweller, van Merriënboer, \& Paas, 1998). Recent theoretical generalizations of the theory (Sweller, 2003, 2004, 2007; Van Merriënboer \& Sweller, 2005) and empirical studies related to the expertise reversal effect (for a recent overview, see Kalyuga, 2007) emphasized the significance of schema-based executive guidance as another, although closely related, role of LTM schemas in learning.

\section{Schema-based executive guidance during knowledge elaboration}

The executive guidance in complex cognitive processes involves planning, organization, and government of mental processes, including engagement and coordination of different cognitive activities and directing learner attention to appropriate information. It is similar to the terms "executive control" and "executive functions" that are used to denote metacognitive regulation of cognition (Brown, 1978, 1987). Metacognition generally refers to the knowledge of the learner's own cognitive processes and the ability to actively control these processes (Flavell, 1976). Metacognitive regulation determines a person's ability to monitor, plan, evaluate, and control the processing of information relevant to the goal.

The above terms are also used in neuropsychological research to denote brain functions such as attention, WM, as well as sequencing, updating, etc. (Fernandez-Duque, Baird, $\&$ Posner, 2000). In theoretical frameworks for the executive control of basic-level cognitive processes, executive functions are usually performed by separate components of our cognitive system (e.g., a central executive in the WM model of Baddeley, 1986; for comprehensive reviews of available frameworks, see Miyake \& Shah, 1999). In empirical studies of executive functions in such frameworks, these functions are usually applied to relatively simple tasks with a very limited (if any) explicit involvement of LTM schemas in consciously controlled cognitive processes. In realistic learning environments, however, we are typically dealing with cognitive tasks that require comprehension and extensive conscious use of complex knowledge structures. In such environments, executive functions or executive control could not be dissociated from the knowledge schemas on which they operate. The absence of a separate independent "regulator" of complex cognition is a principal view of cognitive load theory. It corresponds to an evolutionary approach in CLT that is based on the analogy between the evolution of cognitive and biological systems both of which are considered as examples of natural information-processing systems (Sweller, 2003, 2007). To highlight these knowledge-related characteristics of executive functions in complex cognition, the term "executive guidance" is used throughout this paper. 
If an LTM schema contains automated procedural knowledge, then the processing of the schema-relevant information and subsequent acts are driven by automatic processes that do not require explicit executive guidance or metacognitive regulation. These automated procedures operate without a conscious control and in complex cognitive activities they are usually associated with performing lower-level components. Such implicit knowledge may include the 'learned' automatic structures acquired as a result of extensive practice, as well as products of presumably effortless implicit acquisition of evolutionary-significant structures and heuristics used, for example, in processing space, time, and frequency information (Hasher \& Zacks, 1979) or acquisition of a native language or folk beliefs (Geary, 2007).

In the absence of automated procedures, explicit executive guidance (metacognitive regulation) is required and could be provided by various schemas in LTM. From a metacognitive point of view, these are LTM-based schemas of a declarative nature that represent metacognitive knowledge of tasks, strategies, and goals, and procedural schemas that represent metacognitive skills for orientation, planning, effort allocation, regulation and evaluation of cognitive processes (Efklides, 2001, 2006). These metacognitive knowledge and skills can be used to provide executive guidance, that is, to guide task processing in a manner that implicates executive functions.

When automatic schema-based processing is unavailable or fails for any reason, explicit schemas from LTM acting as metacognitive knowledge and skills can be called in to provide executive guidance for processing the learning task in a conscious and controlled way. For example, when we need to perform a text search, the effective explicit executive guidance could be provided by consciously applying (in WM) metacognitive knowledge of strategies in using higher-level content representation means (e.g., text organizers such as the table of contents or index). In the absence of such knowledge in LTM, the search is likely to be a random activity (Rouet, 2006).

Thus, the executive guidance could be closely associated with metacognition. Even though, according to a traditional view of metacognition, the metalevel of cognition is dissociated from the processes at the cognitive (object) level (Nelson \& Narens, 1994), the metalevel is essentially made up of the schemas developed at the lower cognitive level that are modulated according to a task at hand. Such executive guidance provides flexibility to cognitive processes in the absence of automated schema-based responses (Fernandez-Duque et al., 2000).

Within the theoretical framework of cognitive load theory, it is assumed that the executive guidance during complex cognitive activities, including knowledge construction and elaboration, is provided by available LTM explicit schemas at different levels of specificity rather than separate structural components in our cognitive architecture. The source of executive guidance for high-level cognitive processes is considered as a virtual entity at a metacognitive level that operates on the cognitive level and is constructed for each specific situation or task. This metacognitive level of cognition is not different in nature from cognitive schemas. It is a representation of cognition rather than a separate cognitive structure; a cognitive process that uses knowledge structures to represent cognition (Nelson, 1996) and guide the sequencing of other structures in a top-down manner.

According to this functional approach to executive guidance, appropriate task-relevant schemas in LTM (cognitive level) are combined to manage incoming elements of information (Kalyuga \& Sweller, 2005). During knowledge elaboration, these elements of information are consciously processed in WM where knowledge is actively reconstructed. Available schemas in LTM may effectively guide this reconstruction process. Our higher-level cognition may thus operate as a dynamic system in which the processes of knowledge elaboration are guided by available schemas which, in turn, are updated and modified as a result of such processes.

The concept of long-term working memory (LTWM; Ericsson \& Kintsch, 1995) and theory of skilled memory (Ericsson \& Staszewski, 1989; Kintsch, 1998) provide examples of a possible executive guidance by LTM knowledge base. Schemas associated with elements of information currently active in WM may create an LTWM construct with capacity and duration characteristics that are different from those of WM. These currently active elements of information could represent online task-specific knowledge or other metacognitive experiences (according to Efklides, 2006, 2008) that guide cognitive processing by activating relevant schemas in LTM.

For example, when reading a written text, we construct and continuously update a situation model based on our prior knowledge (e.g., domain knowledge and grammar rules). If the relevant schemas are triggered automatically, then no explicit executive guidance is needed as the subsequent information from the task is simply matched to the situational model. If inconsistencies are detected, then metacognitive knowledge and executive guidance are called in to regulate cognitive processing. The situation model effectively represents the current content of LTWM that directs our attention and regulates the reading process. Because this situation model is sufficiently durable and resistant to temporary interferences, we can resume reading after an interruption without returning to previously read sections and paragraphs (Kintsch, 1998).

Similarly, experienced electrical technicians can fluently read and elaborate a complex wiring diagram using their rich knowledge base of connection patterns for electrical components. In solving algebra equations, available schematic solution procedures and rules in LTM are activated when we approach a task (making up the current content of LTWM) and provide the executive guidance for performing specific solution steps. In our everyday life, we are 'experts' in handling many familiar complex situations, having mastered thousands of classes of context that are recognized, activated and used to govern our everyday activities (Graesser, 2006). Each context is associated with a set of cognitive representations (schemas) that are stored in LTM and provide an executive guidance when activated in a specific situation. In contrast to complex learning tasks, many such schemas in everyday situations are 
automated and operate without conscious control (Bargh \& Chartrand, 1999).

\subsection{Optimizing executive guidance in knowledge elaboration}

In the presence of relevant schemas in LTM, WM can effectively handle rich cognitive activities and organize very complex environments. On the other hand, when no schemas are available or only partly available to provide an executive guidance in a novel situation, we use general search strategies such as means-ends analysis (Newell \& Simon, 1972). For instance, in all the above examples, in the absence of appropriate task-relevant schemas in LTM, we would resort to search processes by trying to fit different behavioral patterns to handle unfamiliar life situations, apply various situational or linguistic schemes to understand the text written in unfamiliar genre or language, or randomly probe specific numbers or operations in trial-and-error attempts to solve an unfamiliar type of algebra equation.

Even if strategies based on search-and-test procedures allow us to eventually reach the problem goals, they are inefficient as learning means because of high levels of wasteful (extraneous to learning goals) WM load that can cause cognitive overload (Sweller, 1988). A better alternative could be offered by external instruction that would provide executive guidance for knowledge construction and elaboration processes. This external instruction would effectively provide a partial substitute for the missing schema-based executive guidance at the initial stages of learning by telling the learners how to handle the situation or solve a task (for example, by explicit directions from an instructor or by presenting a worked example that demonstrates all the appropriate solution steps). From a metacognitive point of view (see Efklides, 2008), this is a metacognitive process that operates at a "social level of metacognition". Even when no instructor is present and worked examples are used instead, the presentation of solution steps and any explanations given or asked presuppose metacognitive awareness of one's own (instructor's) as well as of the students' knowledge.

From this perspective, the role of direct instruction in learning could be interpreted as providing external executive guidance to substitute for missing internal, schema-based guidance in order to reduce extraneous to learning (wasteful) WM load. Determining instructional methods, procedures, and formats that provide optimal executive guidance in different learning situations (e.g., for learners with different levels of prior knowledge) is one of the questions that cognitive load theory attempts to answer. If a major role of instruction is providing a substitute for unavailable LTM schema-based executive guidance, then instructional design may need to follow (or imitate) main characteristics and types of schemas associated with a domain. Schema-based instruction may help students to learn stable information patterns and categorization schemes that would allow them to effectively use appropriate knowledge for dealing with different types of situation without extraneous cognitive load. For example, a schema-based instructional approach in the area of arithmetic word problems was investigated by Marshall (1995). The approach may need to be further explored and extended to different domains.

The relative share of available LTM schemas and required external guidance for a task depends on the level of learner experience in the task domain. While, for beginners, instruction may provide the major source of guidance, for experienced learners, the necessary schemas could be available in LTM (including metacognitive knowledge and strategies for resolving possible conflicts between these schemas). In most learning situations, these two sources of executive guidance need to complement each other. Ideally, a well-balanced executive guidance should be based on LTM schemas when dealing with previously learned information, and on external instructional guidance when dealing with unlearned and relatively new units of information. For example, Clement and Steinberg (2002) provided a detailed description of the complementing roles of learner's increasingly sophisticated internal knowledge (explanatory models) and external tutor's guidance in a case-based study on elaborating knowledge of electric circuits.

Thus, an efficient knowledge elaboration process requires continuous balancing of the executive guidance based on the current state of learner knowledge in a domain. In a situation where no executive guidance is provided for dealing with new information by either of these sources, learners have to resort to general search strategies with the associated extraneous WM load that can cause cognitive overload. This happens, for example, when unguided problem-solving or exploratory learning environments are used with novice learners who do not have any prior knowledge in a domain.

On the other hand, for more advanced learners, there could be an overlap between schema-based automated or conscious metacognitive regulation and instruction-based executive guidance for dealing with the same information and cognitive processes. Therefore, a learner may need to reconcile and cross-reference the related overlapping components of such unbalanced executive guidance consuming additional WM resources and causing extraneous cognitive load. For example, experts may need to turn from automated schema-based processing to consciously controlled processing that requires WM resources. Consequently, less capacity could be available for new knowledge acquisition and elaboration, resulting in the expertise reversal effect (Kalyuga, 2005, 2007; Kalyuga Ayres, Chandler, \& Sweller, 2003). For example, Catrambone and Yuasa (2006) suggested a possible expertise reversal effect when relatively knowledgeable learners were required to generate extended elaborations in a familiar area. Such elaborations could produce excessive levels of unnecessary extraneous cognitive load and interfere with acquisition and automation of relatively new or higher-level schemas.

This explanation of the expertise reversal effect within a cognitive load framework was supported by the patterns of cognitive load measured using subjective rating scales. Higher levels of cognitive load were experienced by advanced learners who studied redundant instructional guidance than by those who studied minimal instructional materials. The 
alternative explanations of the expertise reversal effect, suggested by other researchers, are based on learner motivation and the degree of learner engagement. For example, more experienced learners may not be sufficiently motivated to be deeply engaged in studying seemingly familiar explanations (McNamara, Kintsch, Songer, \& Kintsch, 1996; Paas, Tuovinen, van Merriënboer, \& Darabi, 2005). Establishing the exact nature of the expertise reversal effect will require direct empirical evidence of associated cognitive processes, for example, by analyzing advanced learners' concurrent and retrospective verbal reports supplemented by the records of eye movements when studying well-guided instructional materials in familiar task domains.

\section{Elaborating to higher-level schemas}

Knowledge elaboration processes and the expertise reversal effect in the above discussion were concerned mainly with relatively narrow domain-specific knowledge and skills. Knowledge elaboration in complex learning extends beyond this level and is aimed at the acquisition of higher-level schemas that could be flexibly applied in changing situations. Such schemas may represent generalized domain-specific knowledge structures, as well as metacognitive knowledge and procedures at different levels of generality (including "domain-free" schemas). The successful transfer of acquired knowledge could be possible when this knowledge is elaborated to higher-order schemas. The acquisition of automated specific knowledge and skills schemas related to conditions of their applicability within a domain is important for learning flexible higher-level knowledge and skills in this domain. Within a cognitive load framework, this importance is related to freeing cognitive resources for such learning to occur.

The acquisition of higher-level schemas may be necessary for effective executive guidance in new situations and flexible problem-solving. Therefore, knowledge elaboration at more advanced stages of learning may involve making generalizations on domain-specific schemas, integrating new information into appropriate higher-level schemas, restructuring knowledge at different levels of generality according to a specific task situation and current level of learner knowledge. For example, new knowledge elements can be developed by adapting a higher-level schema to the current situation (e.g., generating examples to illustrate abstract concepts); new knowledge could be integrated into a schema; or generalized knowledge could be modified based on new instances.

Another important feature of higher-level flexible problemsolving skills is the ability to disengage well-learned prior schemas that may not work in new situations. For example, the execution of entrenched (e.g., automated) schemas could be inhibited in favor of consciously applied higher-level strategies or effortful reasoning and problem-solving. In order to withstand high levels of cognitive load involved in such processes, metacognitive knowledge of strategies for monitoring cognitive load and metacognitive skills in allocating and managing cognitive resources are required. For examples, learners may intentionally ignore redundant sources of information or presentation modes that could otherwise overload WM, or intentionally attempt to group ("chunk") incoming elements of new information in order to reduce possible cognitive overload.

The characteristics of learning alter significantly as learners develop more knowledge in a domain. In the absence of relevant schemas, learners may require considerable instructional support to extend their knowledge base. As these learners become more experienced in the domain, they may increasingly rely on retrieving and applying available LTM schemas to handle situations within their areas of experience without overloading WM. This knowledge-based performance of advanced learners makes cognitive resources available for acquisition of higher-order metacognitive knowledge and skills.

Some specific knowledge and skills may need to be developed initially to a high degree of efficiency to free WM for the following higher-level knowledge elaboration (specificto-general approach). On the other hand, higher-level conceptual structures could be acquired at the beginning (the big picture), followed by the specific knowledge elaboration and practice with procedures (general-to-specific approach). Moving from a general structure to its elaboration and back may result in the acquisition of specific knowledge components as parts of whole rather than isolated information (Eylon \& Reif, 1984; Glaser, 1990). For example, the Four-Component Instructional Design Model (4C/ID; Van Merriënboer, 1997) is based on sequences of whole-task practice situations that support the acquisition of generalized declarative and procedural schemas that guide non-recurrent aspects of complex flexible skills. The learning tasks are organized into a simple-to-complex sequence of task classes with gradually diminishing levels of instructional support within each class.

Another possible approach to knowledge elaboration leading to flexible skills could involve moving between different levels of generalization in worked examples with appropriate fading of instructional support. This "vertical" fading represents a different dimension that complements the traditional ("horizontal") fading. The "horizontal" fading occurs at the same level of generalization when parts of worked examples or completion tasks are progressively replaced with problem-solving steps as levels of learner experience increase (Renkl \& Atkinson, 2003; Van Merriënboer, 1990). With the "vertical" fading, students could be presented, for example, with a worked-out general strategic approach (and corresponding conceptual framework) and required to specify it in concrete situations with gradually faded levels of instructional support (e.g., by completing by themselves more steps as problem-solving or exploratory tasks). Learners could also be presented with worked-out specific procedures and required to gradually (with appropriately faded support) figure out general strategic steps involved in the execution of these procedures. Optimal ways of combining the suggested approach with traditional fading and completion strategies in the process of knowledge elaboration need to be investigated. 
Adaptive learning environments that tailor knowledge elaboration processes to changing characteristics of individual learners could provide effective means for managing cognitive load. Tailoring dynamically levels of external instructional guidance to current levels of learner knowledge as they gradually change during learning represents a major instructional implication of the expertise reversal effect. Such tailoring could provide an optimal and well-balanced executive guidance throughout the process of knowledge elaboration. The next section will discuss an approach to designing adaptive learning environments starting from the description of a rapid online method for assessing the learner's LTMbased schemas that can potentially provide executive guidance for knowledge elaboration processes.

\section{Adapting executive guidance to levels of learner knowledge}

Appropriately measuring current levels of knowledge structures in real time is an important means for designing optimal knowledge elaboration processes. As was noted above, LTM schemas guide the processes that take place in WM during complex cognitive activities. Therefore, evaluating sources of executive guidance while learners perform complex tasks may provide indicators of levels of acquisition of corresponding LTM schemas. It is possible to obtain such information using concurrent verbal reports (Kalyuga, 2008). However, this method is very time consuming and not easy to computerize for online use.

In an alternative approach, the required evidence could be obtained by observing what levels of schemas (if any) learners begin applying immediately to briefly presented tasks. Advanced learners may be able to immediately see higherlevel structures in the material, while novices could only see random lower-level components. Available LTM schemas determine such differences. With this first-step diagnostic method, the schemas that guide learners' problem-solving as they approach a task could be established by asking students to rapidly indicate their first step towards solution of the task. Learners with higher levels of knowledge would be better able to retrieve appropriate LTM schemas and rapidly generate advance stages of the solution. This method effectively evaluates schema-based executive guidance structures (if any) a learner uses while approaching a specific task or situation. Different first steps would indicate different levels of acquisition of corresponding schemas in LTM. It should be noted that even though these indicators of schemas are obtained using specific problems, they are independent from the specific learning tasks and relate to schemas as generic concepts.

In a series of validation studies of the first-step diagnostic method in algebra, coordinate geometry, and arithmetic word problems, learners were presented with a test task for a limited time (sufficient for reading and/or inspecting the task statement) and asked to rapidly indicate their first step toward solution. Results indicated significant correlations between performance on these rapid tests (test times were reduced up to five times) and traditional measures of knowledge that required complete solutions of similar tasks (Kalyuga, 2006b; Kalyuga \& Sweller, 2004).

Alternatively, learners could be presented with a series of possible (correct and incorrect) steps corresponding to various stages of the solution procedure, and asked to rapidly verify the suggested steps. This rapid verification method has been recently validated by comparing students' rapid (up to 3.5 times faster) online test scores with results of observations of the same students' problem-solving steps using videorecordings and concurrent verbal reports. Task domains of kinematics and mathematics were used in the study. Results indicated significant correlations between both types of performance measure (Kalyuga, 2008).

In a series of studies with adaptive online tutorials for high school students, the rapid diagnostic methods were used for the dynamic selection of appropriate levels of instructional guidance for learners with different levels of knowledge in specific task domains. Two studies (see Experiment 4 in Kalyuga \& Sweller, 2004, 2005) were conducted in the domain of linear algebra equations, and the third study (Kalyuga, 2006a) used vector-addition motion problems in kinematics. In all three studies, at the beginning of an experimental session, each learner in learner-adapted groups was allocated to an appropriate level of guidance according to the outcome of the initial rapid test. Depending on the outcomes of the rapid probes during instruction, the learner was allowed to proceed to the next stage of the tutorial or was required to repeat the same stage and then take the rapid test again. At each subsequent stage of the tutoring session, less instructional guidance was provided to learners and a higher level of the rapid diagnostic tasks was used at the end of the stage. The tutorials were designed as a series of worked examples, completion assignments, and conventional problems. Kalyuga and Sweller (2005) combined the rapid measures of knowledge with learner subjective ratings of cognitive load (higher levels of proficiency were associated with higher test scores and more effortless performance). The results indicated that both adaptation procedures (one using rapid tests only and another using an efficiency-based measure of proficiency) outperformed non-adaptive instruction formats with no significant differences between the adaptation methods.

Thus, learning was enhanced by adapting knowledge elaboration processes to levels of learner knowledge in specific task domains providing evidence for the importance of balancing executive guidance and individualizing knowledge elaboration procedures. However, the described systemcontrolled adaptive environments may not be the best environments for the development of higher-level self-regulation skills required for flexible performance in changing situations. Alternative adaptive approaches could require combining system and learner controls during advanced learning stages. For example, in a shared control model, the system first selects a subset of tasks based on learner performance scores and cognitive load ratings (a system-controlled component), and then presents this subset to the learner who makes the final decision (a learner-controlled component) (Corbalan, Kester, 
\& van Merriënboer, 2006). The level of learner control could be varied with the development of domain-specific and selfregulation skills. Advisory models may provide learners with adaptive guidance in selecting learning tasks (adaptive guidance approach, Bell \& Kozlowski, 2002).

When solving problems in new situations, experts usually spend more time on a qualitative analysis of the problem, planning, and building explicit representations of the situation before applying specific solution steps (Larkin, 1985; Simon \& Simon, 1978). Therefore, the applicability of rapid methods to diagnosing higher levels of knowledge and skills requires further investigation. It should be focused on learner capabilities of identifying or verifying rapidly generalized conceptual schemas and strategic approaches rather than specific solution steps. For example, if a high-level expert in troubleshooting electronic systems encounters a new situation for which he or she has no available specific solution method, high-level abstract conceptual knowledge of the system may be used for analyzing a possible cause of the malfunction and pinpointing a faulty major functional part of the system. Then, the expert may move to analyzing this part to identify a possible defective lower-level component, and so on. Based on the reported application of high-level knowledge as a first step, it may be possible to rapidly identify such a flexible topdown approach. On the other hand, random trials or search for available specific rules will be reported when a person with a less flexible set of skills first approaches the situation. The immediate actions on approach will reflect the highest available level of organized knowledge and skills. Further research needs to establish optimal combinations of different types of control for developing flexible knowledge and skills in adaptive complex learning environments that use rapid methods for diagnosing higher levels of knowledge.

\section{Conclusion}

According to the main points discussed in this paper, knowledge elaboration processes require executive guidance that is shared between the learner and instructional means (or another expert). Specifically, three processes are identified:

(1) The available knowledge base in learner LTM is used to provide executive guidance in the process of knowledge elaboration;

(2) External instructional guidance substitutes for missing LTM schema-based guidance;

(3) Adaptive learning environments based on rapid diagnostic methods could be effective means for tailoring knowledge elaboration processes to changing characteristics of individual learners and optimizing executive guidance at different stages of knowledge elaboration.

Absence of adequate executive guidance may cause learner cognitive activities that impose wasteful (extraneous to learning) forms of cognitive load and result in WM overload. Therefore, providing adequate executive guidance is associated with avoiding such extraneous cognitive load and preventing potential WM overload. Non-optimal executive guidance could cause excessive levels of cognitive load during knowledge elaboration due to (1) insufficient learner prior knowledge that is not substituted by appropriate instructional guidance, and (2) an overlap between the available knowledge base and provided instructional guidance that are both aimed at the same elaborative cognitive activities. Continuous balancing of executive guidance is essential for reducing or eliminating these sources of cognitive overload by presenting required guidance at the appropriate time and removing unnecessary redundant support as learner proficiency in a domain increases. Adaptive learning environments that dynamically tailor levels of external instructional support to changing individual levels of learner knowledge could effectively optimize executive guidance.

Studies in the expertise reversal effect suggest that for more knowledgeable learners the additional cognitive load (as indicated by subjective rating measures) during knowledge elaboration could be imposed by the process of reconciling related components of available knowledge base and externally provided instructional guidance. Direct empirical evidence of such processes should be obtained in future studies (e.g., by analyzing advanced learners' concurrent verbal reports when studying well-guided instructional materials in familiar task domains). This paper also emphasized that the expertise reversal effect as well as the suggested rapid diagnostic methods were investigated only in relation to taskspecific knowledge and skills. Their applicability to higherlevel knowledge and skills needs to be established in further studies.

Rapid methods of cognitive diagnosis capable of accurately detecting different levels of learner schemas are essential for adaptive learning environments that cannot use traditional diagnostic techniques in real time. Most studies of these methods have been conducted in well-structured task domains with predictable solution paths. Future studies need to investigate the usability of the approach in poorly specified task domains with multiple possible solution routes or with different possible graphical representations of situations. In such task domains, various possible expert-like solution paths need to be established before applying rapid assessment methods. Then, a limited number of selected possible problem states or levels of solution procedure could be included for verification into rapid assessment tasks.

More studies in applying the rapid methods to diagnosing levels of declarative (rather than only procedural) knowledge are also required. For example, expert learners in electronics and electrical engineering may use their declarative knowledge of complex technical systems to immediately see higherlevel integrated structures in such systems and analyze them using a top-down approach. On the other hand, novices can see only some isolated lower-level components. The rapid assessment of knowledge schemas could be based on presenting, for a limited time, a series of diagrammatic representations of selected sub-systems sequenced according to levels of their structural complexity and types of component involved. The participants could be required to identify the 
meaningful systems at a level they can figure out immediately. A learner who has appropriate higher-level declarative schemas for the whole system might be able to determine the overall system immediately. A less knowledgeable person will have to analyze sub-systems or even individual components separately, and at best could identify quickly only the first subjectively meaningful component she/he encounters.

Elaborating higher-level knowledge requires cognitive resources for dealing with flexible, non-routine aspects of performance. Acquisition of task-specific skills is an essential condition for the release of such resources. Based on the finegrained dynamic (real-time) assessment of levels of learner knowledge, adaptive environments could provide learners with individually tailored levels of instructional support for handling relatively new situations without a cognitive overload, thus optimizing executive guidance in knowledge elaboration processes. In future, more comprehensive studies are needed for comparing different dynamic rapid diagnosis-based adaptive methodologies that are optimal for building flexible higher-level knowledge and skills. Optimized shared-control and adaptive-guidance environments need to be developed and tested in complex and less structured domains.

Our understanding of the role of organized knowledge base in learning and changes in knowledge elaboration processes that occur as learners acquire more experience in a task domain have been widened over the last decades. Rapid methods for diagnosing learner knowledge in specific task domains and adaptive procedures for tailoring levels of instructional support to changing individual levels of knowledge have been tested. These developments create conditions for the design of adaptive learning environments that effectively balance executive guidance during knowledge elaboration processes in a dynamic and cognitively optimal way.

\section{Acknowledgements}

I would like to thank the journal's editor, Anastasia Efklides, for her valuable ideas and suggestions for improving the final version of the manuscript.

\section{References}

Anderson, J. R. (1995). Learning and memory: an integrated approach. New York: Wiley.

Anderson, R. C. (1977). Schema-directed processes in language comprehension. In A. Lesgold, J. Pelligrino, S. Fokkema, \& R. Glaser (Eds.), Cognitive psychology and instruction (pp. 67-82). New York: Plenum.

Baddeley, A. D. (1986). Working memory. New York: Oxford University Press.

Bargh, J. A., \& Chartrand, T. L. (1999). The unbearable automaticity of being. American Psychologist, 54, 462-479.

Bartlett, F. C. (1932). Remembering: a study in experimental and social psychology. Cambridge, UK: Cambridge University Press.

Bell, B. S., \& Kozlowski, S. W. J. (2002). Adaptive guidance: enhancing selfregulation, knowledge, and performance in technology-based training. Personnel Psychology, 55, 267-306.

Brown, A. L. (1978). Knowing when, where, and how to remember: a problem of metacognition. In R. Glaser (Ed.), Advances in instructional psychology, Vol. 1 (pp. 77-165). Hillsdale, NJ: Erlbaum.
Brown, A. L. (1987). Metacognition, executive control, self-regulation and other more mysterious mechanisms. In F. Weinert, \& R. Kluwe (Eds.), Classroom management (pp. 144-181). Hillsdale, NJ: Erlbaum.

Catrambone, R., \& Yuasa, M. (2006). Acquisition of procedures: the effects of example elaborations and active learning exercises. Learning and Instruction, 16, 139-153.

Chi, M. T. H., Bassok, M., Lewis, M. W., Reinmann, P., \& Glaser, R. (1989). Self-explanations: how students study and use examples in learning to solve problems. Cognitive Science, 13, 145-182.

Chi, M., Glaser, R., \& Rees, E. (1982). Expertise in problem solving. In R. Sternberg (Ed.), Advances in the psychology of human intelligence (pp. 7-75). Hillsdale, NJ: Erlbaum.

Clement, J. J., \& Steinberg, M. S. (2002). Step-wise evolution of mental models of electric circuits: a "learning-aloud" case study. The Journal of the Learning Sciences, 11, 389-452.

Corbalan, G., Kester, L., \& van Merriënboer, J. J. G. (2006). Towards a personalized task selection model with shared instructional control. Instructional Science, 34, 399-422.

Cowan, N. (2001). The magical number 4 in short-term memory: a reconsideration of mental storage capacity. The Behavioral and Brain Sciences, 24, 87-114.

Efklides, A. (2001). Metacognitive experiences in problem solving; cognition, affect, and self-regulation. In A. Efklides, J. Kuhl, \& R. Sorrentino (Eds.), Trends and prospects in motivation research (pp. 297-323). Dordrecht, The Netherlands: Kluwer.

Efklides, A. (2006). Metacognition and affect: what can metacognitive experiences tell us about the learning process? Educational Research Review, 1, $3-14$.

Efklides, A. (2008). Metacognition: defining its facets and levels of functioning in relation to self- and co-regulation. European Psychologist, 13, 277-287.

Ericsson, K. A., \& Kintsch, W. (1995). Long-term working memory. Psychological Review, 102, 211-245.

Ericsson, K. A., \& Staszewski, J. J. (1989). Skilled memory and expertise: mechanisms of exceptional performance. In D. Klahr, \& K. Kotovsky (Eds.), Complex information processing: the impact of Herbert A. Simon (pp. 235-267). Hillsdale, NJ: Erlbaum.

Eylon, B. S., \& Reif, F. (1984). Effects of knowledge organization on task performance. Cognition and Instruction, 1, 5-44.

Fernandez-Duque, D., Baird, J. A., \& Posner, M. I. (2000). Executive attention and metacognitive regulation. Consciousness and Cognition, 9, 288-307.

Flavell, J. H. (1976). Metacognitive aspects of problem solving. In L. B. Resnick (Ed.), The nature of intelligence (pp. 231-235). Hillsdale, NJ: Erlbaum.

Geary, D. (2007). Educating the evolved mind: conceptual foundations for an evolutionary educational psychology. In J. S. Carlson, \& J. R. Levin (Eds.), Psychological perspectives on contemporary educational issues (pp. 199). Greenwich, CT: Information Age Publishing.

Glaser, R. (1990). The reemergence of learning theory within instructional research. American Psychologist, 45, 29-39.

Graesser, A. (2006). Views from a cognitive scientist: cognitive representations underlying discourse are sometimes social. Discourse Studies, 8 , $59-66$.

Hasher, L., \& Zacks, R. T. (1979). Automatic and effortful processes in memory. Journal of Experimental Psychology: General, 108(3), 356-388.

Kalyuga, S. (2005). Prior knowledge principle. In R. Mayer (Ed.), Cambridge handbook of multimedia learning (pp. 325-337). New York: Cambridge University Press.

Kalyuga, S. (2006a). Assessment of learners' organized knowledge structures in adaptive learning environments. Applied Cognitive Psychology, 20, $333-342$.

Kalyuga, S. (2006b). Rapid cognitive assessment of learners' knowledge structures. Learning and Instruction, 16, 1-11.

Kalyuga, S. (2007). Expertise reversal effect and its implications for learnertailored instruction. Educational Psychology Review, 19, 509-539.

Kalyuga, S. (2008). When less is more in cognitive diagnosis: a rapid online method for diagnosing learner task-specific expertise. Journal of Educational Psychology, 100, 603-612. 
Kalyuga, S., Ayres, P., Chandler, P., \& Sweller, J. (2003). Expertise reversal effect. Educational Psychologist, 38, 23-31.

Kalyuga, S., \& Sweller, J. (2004). Measuring knowledge to optimize cognitive load factors during instruction. Journal of Educational Psychology, 96, 558-568.

Kalyuga, S., \& Sweller, J. (2005). Rapid dynamic assessment of expertise to improve the efficiency of adaptive e-learning. Educational Technology, Research and Development, 53, 83-93.

Kintsch, W. (1998). Comprehension: a paradigm for cognition. New York: Cambridge University Press.

Kotovsky, K., Hayes, J. R., \& Simon, H. A. (1985). Why are some problems hard? Evidence from Tower of Hanoi. Cognitive Psychology, 17, 248-294.

Larkin, J. H. (1985). Understanding, problem representation, and skill in physics. In S. F. Chipman, J. W. Segal, \& R. Glaser (Eds.), Thinking and learning skills: research and open questions, Vol. 2 (pp. 141-160). Hillsdale, NJ: Erlbaum.

Marshall, S. (1995). Schemas in problem solving. New York: Cambridge University Press.

Mayer, R. E. (1984). Aids to text comprehension. Educational Psychologist, $19,30-42$.

McNamara, D., Kintsch, E., Songer, N. B., \& Kintsch, W. (1996). Are good texts always better? Interactions of text coherence, background knowledge, and levels of understanding in learning from text. Cognition and Instruction, 14, 1-43.

Miller, G. A. (1956). The magical number seven, plus or minus two: some limits on our capacity for processing information. Psychological Review, 63, 81-97.

Miyake, A., \& Shah, P. (Eds.). (1999). Models of working memory: mechanisms of active maintenance and executive control. Cambridge, England: Cambridge University Press.

Nelson, T. O. (1996). Consciousness and metacognition. American Psychologist, 51, 102-116.

Nelson, T. O., \& Narens, L. (1994). Why investigate metacognition? In J. Metcalfe, \& A. Shimamura (Eds.), Metacognition: knowing about knowing (pp. 1-25) Cambridge, MA: Bradford Books.

Newell, A., \& Simon, H. A. (1972). Human problem solving. Englewood Cliffs, NJ: Prentice-Hall.

Paas, F., Tuovinen, J. E., van Merriënboer, J. J. G., \& Darabi, A. A. (2005). A motivational perspective on the relation between mental effort and performance. Educational Technology, Research and Development, 53, 25-34.

Pressley, M. (1982). Elaboration and memory development. Child Development, 53, 296-309.
Reigeluth, C. M., Merrill, M. D., Wilson, B. G., \& Spiller, R. T. (1980). The elaboration theory of instruction: a model for structuring instruction. Instructional Science, 9(3), 195-219.

Renkl, A., \& Atkinson, R. K. (2003). Structuring the transition from example study to problem solving in cognitive skills acquisition: a cognitive load perspective. Educational Psychologist, 38, 15-22.

Rouet, J.-F. (2006). The skills of document use: from text comprehension to Web-based learning. Mahwah, NJ: Erlbaum.

Rumelhart, D. E. (1980). Schemata: the building blocks of cognition. In R. J. Spiro, B. C. Bruce, \& W. E. Brewer (Eds.), Theoretical issues in reading comprehension (pp. 33-58). Hillsdale, NJ: Erlbaum.

Shiffrin, R., \& Schneider, W. (1977). Controlled and automatic human information processing: II. Perceptual learning, automatic attending, and a general theory. Psychological Review, 84, 127-190.

Simon, D. P., \& Simon, H. A. (1978). Individual differences in solving physics problem. In R. Siegler (Ed.), Children's thinking: what develops (pp. 325348). Hillsdale, NJ: Erlbaum.

Stark, R., Mandl, H., Gruber, H., \& Renkl, A. (2002). Conditions and effects of example elaboration. Learning and Instruction, 12, 39-60.

Sweller, J. (1988). Cognitive load during problem solving: effects on learning. Cognitive Science, 12, 257-285.

Sweller, J. (2003). Evolution of human cognitive architecture. In B. Ross (Ed.), The psychology of learning and motivation, Vol. 43 (pp. 215-266). San Diego, CA: Academic.

Sweller, J. (2004). Instructional design consequences of an analogy between evolution by natural selection and human cognitive architecture. Instructional Science, 32, 9-31.

Sweller, J. (2007). Evolutionary biology and educational psychology. In J. S. Carlson, \& J. R. Levin (Eds.), Psychological perspectives on contemporary educational issues (pp. 165-175). Greenwich, CT: Information Age Publishing.

Sweller, J., van Merriënboer, J., \& Paas, F. (1998). Cognitive architecture and instructional design. Educational Psychology Review, 10, 251-296.

Van Merriënboer, J. J. G. (1990). Strategies for programming instruction in high school: Program completion vs. program generation. Journal of Educational Computing Research, 6, 265-287.

Van Merriënboer, J. J. G. (1997). Training complex cognitive skills: a fourcomponent instructional design model for technical training. Englewood Cliffs, NJ: Educational Technology Publications.

Van Merriënboer, J., \& Sweller, J. (2005). Cognitive load theory and complex learning: recent developments and future directions. Educational Psychology Review, 17, 147-177. 